\title{
White matter hyperintensities and medial temporal lobe atrophy in clinical subtypes of mild cognitive impairment: the DESCRIPA study
}

\author{
$\mathrm{L} A$ van de Pol, ${ }^{1} \mathrm{~F}$ Verhey, ${ }^{2} \mathrm{G}$ B Frisoni, ${ }^{3} \mathrm{M}$ Tsolaki, ${ }^{4} \mathrm{P}$ Papapostolou, ${ }^{5} \mathrm{~F}$ Nobili, ${ }^{6}$ \\ L-O Wahlund, ${ }^{7}$ L Minthon, ${ }^{8}$ L Frölich, ${ }^{9}$ H Hampel, ${ }^{10} \mathrm{H}$ Soininen, ${ }^{11} \mathrm{D} \mathrm{L} \mathrm{Knol,},{ }^{12} \mathrm{~F}$ Barkhof, ${ }^{13}$ \\ P Scheltens, ${ }^{1}$ P J Visser ${ }^{2}$
}

For numbered affiliations see end of article

\section{Correspondence to:}

Dr Laura A van de Pol, VUMC Department of Neurology, PO Box 7057, 1007 MB Amsterdam, The Netherlands: L.vandepol@vumc.nl

Received 21 August 2008 Revised 19 May 2009 Accepted 21 May 2009 Published Online First 18 June 2009

\begin{abstract}
Background: Clinical subtypes of mild cognitive impairment (MCI) may represent different underlying aetiologies.

Methods: This European, multicentre, memory clinic based study (DESCRIPA) of non-demented subjects investigated whether $\mathrm{MCl}$ subtypes have different brain correlates on $\mathrm{MRI}$ and whether the relation between subtypes and brain pathology is modified by age. Using visual rating scales, medial temporal lobe atrophy (MTA) (0-4) and white matter hyperintensities (WMH) (0-30) were assessed.
\end{abstract}

Results: Severity of MTA differed between MCl subtypes $(p<0.001)$, increasing from a mean of 0.8 (SD 0.7) in subjective complaints $(n=77)$ to $1.3(0.8)$ in nonamnestic $\mathrm{MCl}(\mathrm{n}=93)$, and from $1.4(0.9)$ in single domain amnestic $\mathrm{MCl}(\mathrm{n}=70)$ to $1.7(0.9)$ in multiple domain amnestic $\mathrm{MCl}(\mathrm{n}=89)$. The association between $\mathrm{MCl}$ subtype and MTA was modified by age and mainly present in subjects $>70$ years of age. Severity of $\mathrm{WMH}$ did not differ between $\mathrm{MCl}$ subtypes $(\mathrm{p}=0.21)$. However, the combination of MTA and WMH differed between $\mathrm{MCl}$ subtypes ( $p=0.02$ )

Conclusion: We conclude that $\mathrm{MCl}$ subtypes may have different brain substrates, especially in older subjects. Isolated MTA was mainly associated with amnestic $\mathrm{MCl}$ subtypes, suggesting AD as the underlying cause. In nonamnestic $\mathrm{MCl}$, the relatively higher prevalence of MTA in combination with WMH may suggest a different pathophysiological origin.

Mild cognitive impairment (MCI) refers to cognitive decline in non-demented subjects and is considered to be a risk state for Alzheimer's disease (AD) $\cdot^{1-3}$ However, not all subjects with MCI will develop $\mathrm{AD}$-some will remain stable or even improve over time while others will develop other types of dementia. ${ }^{4-6}$ Clinical subtypes of $\mathrm{MCI}$ have been suggested that are assumed to represent different underlying aetiologies. These subtypes are based on the cognitive domains in which the impairment occurs (amnestic versus non-amnestic) and the number of cognitive domains affected (single versus multiple). ${ }^{5}$ The amnestic type of $\mathrm{MCI}$ is regarded as a prodromal form of $\mathrm{AD}$ whereas the subtypes with impairment in nonmemory domains are assumed to represent prodromal stages of other types of dementia, such as vascular dementia or dementia with Lewy bodies. ${ }^{5}$ Preliminary studies have shown that
MCI subtypes may indeed represent different disorders. $^{78}$

Several studies have investigated underlying brain pathology in MCI subtypes, using MRI. In all studies, subjects with single domain or multiple domain amnestic MCI were characterised by medial temporal lobe atrophy (MTA). MTA on $\mathrm{MRI}$ is a sensitive diagnostic marker for $\mathrm{AD} \cdot{ }^{9-12} \mathrm{The}$ presence of MTA in amnestic MCI has been shown to be predictive of $\mathrm{AD} .{ }^{6}{ }^{13}{ }^{14}$ In non-amnestic MCI, conflicting findings have been reported. One study reported MTA to be present in non-amnestic $\mathrm{MCI}^{3}$ while another study did not find this. ${ }^{15}$ White matter hyperintensities (WMH) were more common in non-amnestic MCI compared with amnestic $\mathrm{MCI}^{16}$ although this was not found in another study. ${ }^{17} \mathrm{WMH}$, which are generally viewed as evidence of small vessel disease, are commonly observed on MRI across the cognitive spectrum. ${ }^{17-22}$ No studies have yet investigated the interaction between MTA and WML in subtypes of MCI. This interaction might be of interest as WML and MTA may have an additive effect on cognitive function. ${ }^{23}$

In order to provide further support for the idea that the subclassification of MCI may differentiate between various underlying aetiologies, we investigated the presence of MTA and WMH, and their combination, across MCI subtypes. Subjects were classified into four groups: (1) subjective complaints; (2) non-amnestic MCI; (3) single domain amnestic MCI; and (4) multiple domain amnestic MCI, based on neuropsychological test performance. We hypothesised that MTA would be mainly associated with amnestic MCI whereas WMH might be more involved in non-amnestic MCI. We also investigated whether the prevalence of MTA and WMH in MCI subtypes was dependent on age, educational level and sex, because population based studies have shown that older age, female sex and low educational level are associated with an increased risk for dementia. ${ }^{24}$

\section{METHODS}

\section{Study design}

Subjects were selected from the DESCRIPA study (www.descripa.eu), a multicentre study of the European Alzheimer's Disease Consortium, aiming to develop clinical criteria and screening guidelines for $\mathrm{AD}$ in the pre-dementia stage. A detailed outline of the DESCRIPA study has been described earlier. ${ }^{25}$ Inclusion criteria were: age 55 years or 
older, new referral for the evaluation of cognitive complaints and no diagnosis of dementia. Exclusion criteria were: any somatic, psychiatric or neurological disorder that may have caused the cognitive impairment such as a cerebrovascular accident or strategic infarction with an acute onset of the cognitive impairment, neurodegenerative diseases such as Parkinson's disease, severe head trauma, brain tumour, a history of alcohol abuse and severe depression. The study closely followed regular clinical practice or was performed as part of a research project. For the present study, subjects were selected from 10 centres in which MRI scanning was part of clinical practice or a research project $(n=512)$. MRI was available for 351 (69\%) subjects. Reasons for no MRI included: contraindication for MRI, patient refusal, poor quality of MRI scan and avoidance of waiting lists for MRI assessment. Subjects with and without MRI did not differ with respect to demographic characteristics, score on the Mini-Mental State Examination $(\mathrm{MMSE})^{26}$ or prevalence of vascular risk factors.

Twenty-two subjects who could not be classified as one of the MCI subtypes because of missing data for one or more neuropsychological tests were excluded. These subjects scored lower on MMSE (mean (SD)): 25 (4) $(t=-5.0, \mathrm{df}=346$, $p<0.001$, MMSE scores were missing for one of these 22 subjects and for two subjects of the final sample) and a lower prevalence of hyperlipidaemia $\left(14 \% ; \chi^{2}=4.8, \mathrm{df}=1, \mathrm{p}=0.03\right)$ compared with the rest of the cohort. There were no differences with respect to age, level of education and prevalence of other risk factors. The final study sample consisted of 329 subjects. The study was approved by the local medical ethics committee in each centre.

\section{Baseline clinical assessment}

All subjects underwent a standard battery of examinations, including a clinical history, medical and neurological examination, laboratory tests, functional evaluation using the Clinical Dementia Rating scale, ${ }^{27}$ rating scales for depression and neuropsychiatric symptoms, a neuropsychological test battery (see below) and neuroimaging. General cognition was assessed using the MMSE. The following vascular risk factors were included in the analyses: hypertension (systolic blood pressure $>140 \mathrm{~mm} \mathrm{Hg}$ or diastolic blood pressure $>90 \mathrm{~mm} \mathrm{Hg}$, a history of hypertension or use of antihypertensive medication), a history of diabetes mellitus, hyperlipidaemia and atherosclerosis (a history of ischaemic heart disease, carotid stenosis, cerebrovascular incident or transient ischaemic attack in the past).

\section{Neuropsychological examination}

In each centre, a battery of neuropsychological tests was performed to assess cognitive performance in the domains of memory, language, executive function and attention, and visuoconstruction. The tests used to assess each domain could vary between centres. Raw scores were converted to age, education and gender corrected $z$ scores according to locally collected normative data or published normative data and these z scores were used for further analysis.

\section{Definition of $\mathrm{MCl}$ subtypes}

Subjects were classified into four MCI subtypes on the basis of the performance on tests in the cognitive domains of memory, language, executive function and attention, and visuoconstruction, as described below. Subjects without impairment in any domain were classified as subjective complaints, subjects with impairment in one or more non-memory domains as non-amnestic MCI, subjects with isolated impairment in the memory domain as single domain amnestic MCI and subjects with impairment in the memory domain and at least one other domain as multiple domain amnestic MCI. Impairment was defined as a $z$ score of -1.5 or lower, which equals a score of 1.5 SD below the average score of healthy control subjects after correction for age, sex and education.

Due to variability in the neuropsychological test protocol, the tests used to define MCI subtypes varied between centres. We selected in each centre one test for each domain that was identical or similar to tests used in other centres. The tests to assess memory were the learning measure and delayed recall measure of the Rey Auditory Verbal Learning Test (six centres), ${ }^{28}$ the word list of the Consortium to Establish a Registry for $\mathrm{AD}$ (CERAD) neuropsychological battery (three centres ${ }^{29}$ and the Selective Reminding Test (one centre). ${ }^{30}$ The tests to assess language were 1 min verbal fluency for animals (nine centres) and 1 min verbal fluency for fruits, animals or car trades (one centre). ${ }^{31}$ The test to assess executive function and attention was the Trail Making Test part A and B (all centres). ${ }^{32}$ The tests to assess visuoconstruction were the copy subtest of the Rey-Osterrieth complex figure (six centres), ${ }^{33}$ the copy of the CERAD figures (three centres) ${ }^{29}$ or the copy of figures from the Mental Deterioration Battery (one centre). ${ }^{34}$

Thirty-two subjects classified as non-amnestic MCI (34\% of all subjects in this subgroup) had impairments in more than one non-memory domain and could be considered to have multiple domain non-amnestic MCI. ${ }^{5}$ Post-hoc analyses showed no statistically significant differences with regard to MRI characteristics between subjects with single and multiple domain non-amnestic MCI, and therefore we analysed the data of subjects with non-amnestic MCI as a single group.

\section{MRI acquisition}

All subjects were studied by MRI within a mean of 0.1 (SD 0.2 ) years of the baseline clinical assessment. At each site, subjects were scanned according to the routine MRI protocol, and consequently the scanners and protocols at different sites varied. All scanning was performed at 1.0 or $1.5 \mathrm{~T}$ and included a three-dimensional T1 weighted gradient echo sequence and a fast fluid attenuated inversion recovery (FLAIR) sequence. MRI data were collected and analysed centrally. Sagittal threedimensional T1 weighted images were reformatted in a plane perpendicular to the long axis of the (left) hippocampus (at a slice thickness of $2 \mathrm{~mm}$ ).

\section{Visual rating of MTA and WMH}

MTA was rated on coronal T1 weighted images using a 5 point visual rating scale, ${ }^{12}$ ranging from 0 (no atrophy) to 4 (severe atrophy) based on the height of the hippocampal formation and the surrounding CSF spaces. In the analysis, the average score of left and right was used, as well as the dichotomised score (MTA $\geqslant 2$ = atrophy). The degree of WMH severity was rated on the axial FLAIR images using the Age Related White Matter Changes scale (ARWMC). ${ }^{35}$ Here we used the total degree of WMH (range 0-30) by adding the region specific scores of both hemispheres and a dichotomised score (at the population mean WMH score: $\mathrm{ARWMC}>5=$ moderate $\mathrm{WMH}$ ). All visual ratings were carried out centrally by a single rater (LvdP) who was blinded to the clinical information. The intra-rater agreement for the MTA scale was good (kappa 0.68) as well as the intra-rater agreement for the ARWMC scale, as determined on a test set of 20 MR scans scored twice (weighted kappa 0.95). 
Table 1 Baseline characteristics in the total sample and according to the four $\mathrm{MCl}$ subtypes

\begin{tabular}{|c|c|c|c|c|c|c|c|}
\hline & \multirow{3}{*}{$\begin{array}{l}\text { Total sample } \\
(\mathrm{n}=329)\end{array}$} & \multirow{3}{*}{$\begin{array}{l}\begin{array}{l}\text { Subjective } \\
\text { complaints } \\
(\mathrm{n}=77)\end{array} \\
\text { Group } 1\end{array}$} & \multirow{3}{*}{$\begin{array}{l}\text { Non-amnestic } \\
\mathrm{MCl}(\mathrm{n}=93) \\
\text { Group } 2\end{array}$} & \multicolumn{2}{|l|}{ Amnestic $\mathrm{MCl}$} & \multirow{3}{*}{$\begin{array}{l}\text { Overall } \\
\text { p Value }\end{array}$} & \multirow{3}{*}{$\begin{array}{l}\text { Group } \\
\text { comparisons }\end{array}$} \\
\hline & & & & $\begin{array}{l}\text { Single domain } \\
(n=70)\end{array}$ & $\begin{array}{l}\text { Multiple domain } \\
(\mathrm{n}=89)\end{array}$ & & \\
\hline & & & & Group 3 & Group 4 & & \\
\hline \multicolumn{8}{|l|}{ Demographics } \\
\hline Age (years) & $69(8)$ & $66(7)$ & $70(8)$ & $69(8)$ & $71(8)$ & $<0.0001$ & $1<2,4$ \\
\hline Sex (n (\%) female) & $188(57)$ & $39(51)$ & $64(69)$ & $31(44)$ & $54(61)$ & 0.003 & $2>3$ \\
\hline ApoE4 genotype $(\mathrm{n}(\%))^{*}$ & $126(48)$ & $27(45)$ & $27(35)$ & $34(58)$ & $28(41)$ & 0.11 & - \\
\hline \multicolumn{8}{|l|}{ Vascular risk factors } \\
\hline Hypertension (n (\%)) & $216(66)$ & $48(63)$ & $62(67)$ & $45(65)$ & $61(69)$ & 0.95 & - \\
\hline Diabetes mellitus (n (\%)) & $38(12)$ & 7 (9) & $12(13)$ & $4(6)$ & $15(17)$ & 0.28 & - \\
\hline Hyperlipidaemia (n (\%)) & $122(38)$ & $21(28)$ & $36(40)$ & $27(40)$ & $38(44)$ & 0.30 & - \\
\hline Atherosclerosis (n (\%)) & $45(15)$ & $8(11)$ & $13(15)$ & $12(18)$ & $12(15)$ & 0.45 & - \\
\hline Moderate WMH (n (\%)) & $105(32)$ & $20(26)$ & $36(34)$ & $22(31)$ & $27(30)$ & 0.16 & - \\
\hline
\end{tabular}

Values are expressed as mean (SD) unless stated otherwise. ANOVA or logistic regression models for dichotomous outcome variables with age, sex and centre of origin as covariates were performed. Bonferonni correction was used to correct for multiple comparisons.

${ }^{*}$ ApoE genotype was available for 265 subjects: 60 subjective, 77 non-amnestic, 59 amnestic single domain and 69 amnestic multiple domain. Only significant differences are reported.

MCl, mild cognitive impairment; MMSE, Mini-Mental State Examination (0-30); MTA, medial temporal lobe atrophy; WMH, white matter hyperintensities (0-30), MTA present: score $\geqslant 2 ;$ WMH moderate: score $>5$.

\section{APOE}

APOE genotype was determined in a subset of subjects $(n=265$, $81 \%$ ) on genomic DNA extracted from EDTA anticoagulated blood using the PCR technique. Subjects with data on APOE genotype had a higher score on the MMSE compared with subjects with data on APOE genotype (28.1 vs $27.5 ; \mathrm{p}=0.05$ ) while age, years of education, gender, systolic blood pressure, severity of MTA and WMH, and scores on the cognitive tests did not differ between the groups.

\section{Statistics}

SPSS for Windows, V.12.0 (Chicago, Illinois, USA) was used for data analysis. Characteristics of subjects in the present study sample were compared with characteristics of excluded subjects without MRI and missing neuropsychological data using Student's t tests or $\chi^{2}$ tests when appropriate. Subsequently, group differences between the MCI subtypes were assessed using analysis of variance (ANOVA) or logistic regression models for dichotomous outcome variables. Age and sex were used as covariates and centre of origin as a categorical covariate. Bonferroni correction was used to adjust for multiple comparisons in the post hoc pairwise comparisons. MTA and WMH scores were used both in their continuous as well as dichotomised form (as described in the visual rating section above). Interactions between MCI subtype and age (dichotomised at 70 years), gender and level of education were tested for severity of MTA and WMH (as continuous variables).

Finally, using both dichotomised MRI measures we computed a new MTA-WMH categorical variable, yielding four groups: (1) MTA and WMH absent; (2) MTA absent, WMH present; (3) MTA present, WMH absent; and (4) MTA and WMH present. The difference in distribution of subjects over the MTA-WMH
Figure 1 Bar chart showing the severity of medial temporal lobe atrophy (MTA) in each mild cognitive impairment (MCl) subtype by age group $(<70$ vs $\geqslant 70$ years). Numbers indicate mean MTA score (and number of subjects).

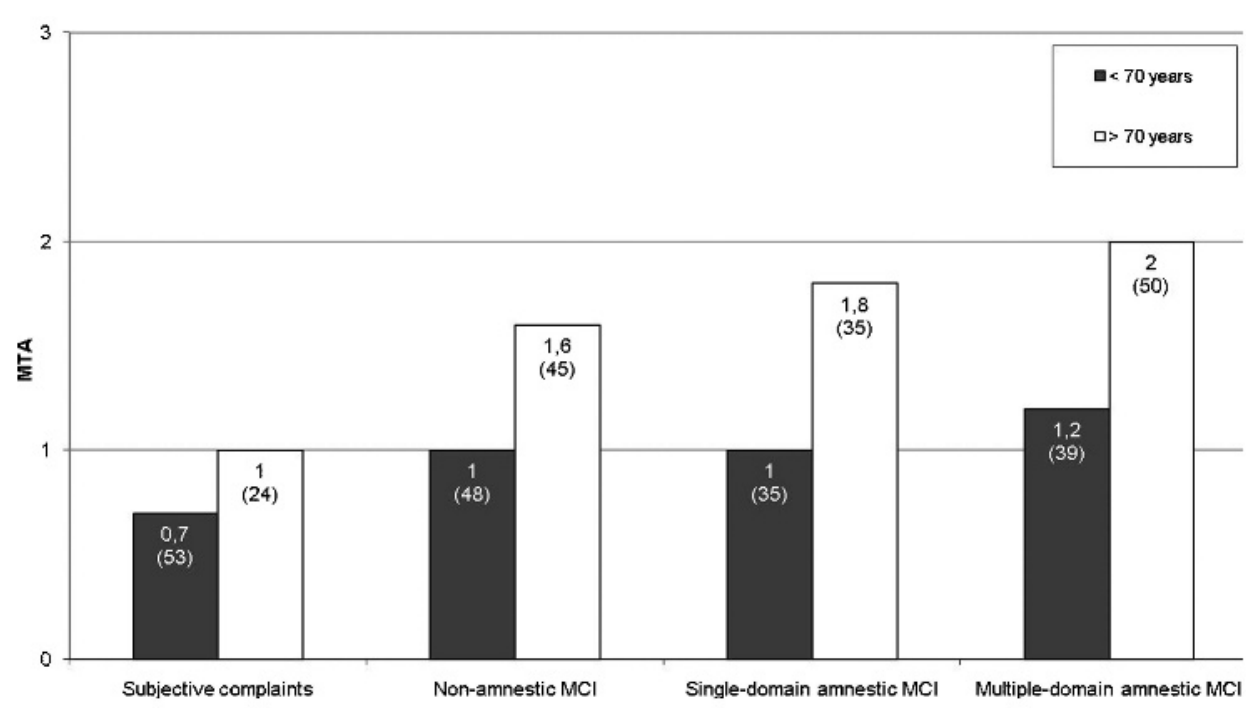


groups across MCI subtypes was assessed using multinomial logistic regression analysis with MTA-WMH group as the dependent variable and MCI subtype as a predictor, adjusting for age.

\section{RESULTS}

Table 1 shows the baseline characteristics for the total study sample and for the four MCI subtypes. Age $(p<0.0001)$, sex $\left(\chi^{2}=36.1, \mathrm{df}=13, \mathrm{p}=0.028\right)$, years of education $(\mathrm{p}=0.027)$ and MMSE scores $(\mathrm{Fp}<0.0001)$ were significantly different between the groups. There were no differences in APOE genotype or the prevalence of vascular risk factors across the MCI subtypes.

Severity of MTA, as reflected by the continuous MTA score, differed across the MCI subtypes $(p<0.001)$. Pairwise comparisons showed that subjects with subjective complaints had less severe MTA than subjects with non-amnestic MCI $(p=0.047)$, single domain amnestic MCI $(p=0.005)$ and multiple domain amnestic MCI ( $p<0.0001)$, and that subjects with non-amnestic MCI had less severe MTA compared with subjects with multiple domain amnestic MCI $(p=0.006)$. The presence of MTA $\geqslant 2$ increased from $14 \%$ of subjects with subjective complaints to $38 \%$ of subjects with non-amnestic MCI, to $44 \%$ in subjects with single domain amnestic MCI and to $55 \%$ in subjects with multiple domain amnestic MCI (post hoc pairwise comparisons: subjective complaints versus all other subtypes, $\mathrm{p}<0.01$ ). In contrast, the total ARWMC score, and the proportion of subjects with at least moderate WMH (ARWMC score $>5$ ), did not differ across the MCI subtypes.

Correction of the analyses for level of education and vascular risk factors did not essentially change the results (data not shown).

The association between the MTA score and MCI subtype was modified by age, as indicated by a significant interaction between age (dichotomised at 70 years) and subtype $(p=0.03)$. If age was used as a continuous variable, the effect of age on MTA was also statistically significant. Post hoc analysis showed that in older subjects, MTA score was strongly associated with MCI subtype $(p<0.0001)$, in contrast with a weaker association in younger subjects $(p=0.02)$ (fig 1$)$.

No significant interaction between MCI subtype and age group in association with WMH was present $(p=0.19)$, nor were there any interactions for gender or level of education and MCI subtype in association with severity of MTA ( $p=0.53$ and $\mathrm{p}=0.70)$ or $\mathrm{WMH}(\mathrm{p}=0.74$ and $\mathrm{p}=0.31)$.

The distribution of four MRI categories, based on the dichotomised scores of MTA (0-1 vs $\geqslant 2)$ and WMH (0-5 vs $\geqslant 6)$, over the MCI subtypes is shown in fig 2. A multinomial logistic regression model, adjusted for age, showed that the distribution of subjects over the four MRI categories differed between the four MCI subtypes (overall: $\chi^{2}=25.7 \mathrm{df}=9$, $\mathrm{p}=0.002$, Nagelkerke's $\left.\mathrm{R}^{2}=0.30\right)$. Pairwise comparisons showed that the distribution of subjects over the four MRI categories differed between subjects with subjective complaints and the amnestic MCI subtypes $(p=0.023$ for single domain amnestic MCI and $p=0.002$ for multiple domain amnestic $\mathrm{MCI}$ ) and between non-amnestic MCI and multiple domain amnestic MCI $(p=0.015)$. In more detail, in multiple domain amnestic MCI, a greater proportion of subjects had isolated MTA versus MTA in combination with WMH compared with subjects with non-amnestic MCI $(p=0.011)$. Subjects with subjective complaints had a greater proportion of subjects with isolated WMH versus isolated MTA compared with multiple domain amnestic MCI $(p=0.002)$. Finally, the proportion of subjects without any MRI abnormality compared with isolated MTA was greater in subjective complaints compared with both types of amnestic MCI ( $p=0.004$ and $p<0.0001$, respectively), and in non-amnestic MCI versus multiple domain amnestic MCI $(p=0.003)$. Other pairwise comparisons were not statistically significant.

\section{DISCUSSION}

We have provided evidence that the clinical subtypes of $\mathrm{MCI}$, based on neuropsychological profiles, may have different brain substrates. Both single and multiple domain amnestic MCI were associated with more severe MTA in comparison with subjects with subjective complaints. This is in keeping with previous studies demonstrating atrophy of the medial temporal lobe in subjects with amnestic $\mathrm{MCI}^{36}{ }^{37}$ and may be associated with an increased risk of development of $\mathrm{AD} .{ }^{6}{ }^{14}$ However, MTA was not restricted to amnestic MCI, as a substantial part of subjects with non-amnestic MCI showed MTA as well. This is in line with previous longitudinal studies that have shown that a proportion of subjects with non-amnestic MCI progresses to
Figure 2 Bar chart showing the distribution of MRI subgroups, based on the presence of medial temporal lobe atrophy (MTA) and white matter hyperintensities (WMH), within the mild cognitive impairment (MCI) subtypes. Numbers indicate percentage of subjects (and number of subjects) within each $\mathrm{MCl}$ subtype.

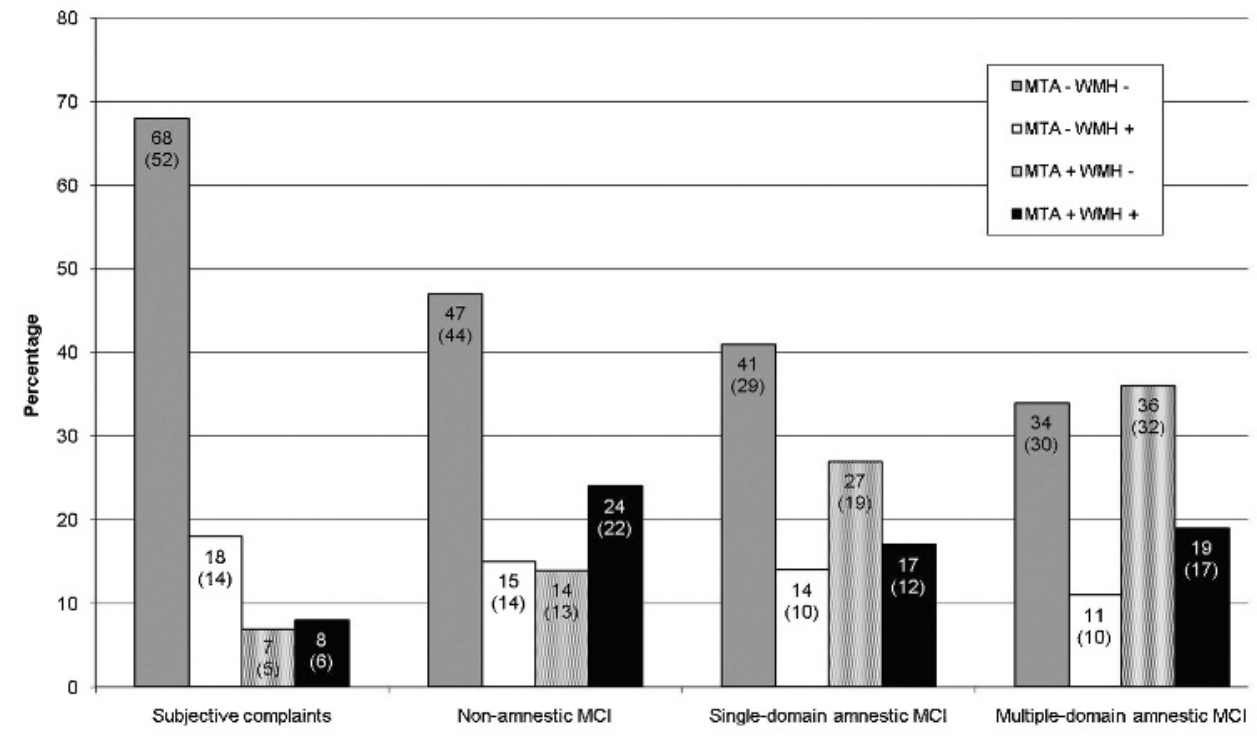


clinical $\mathrm{AD},{ }^{73}$ and another MRI study, using voxel based morphometry, showing varying involvement of the medial temporal lobe in the non-amnestic (single and multiple domain) MCI subtypes. ${ }^{3}$ Alternatively, MTA in non-amnestic MCI may have a different aetiology and relate to vascular causes.

Our findings are in keeping with a study demonstrating that subjects with amnestic MCI had a higher prevalence of MTA than subjects with non-amnestic MCI. ${ }^{15}$ Two other studies showed focal atrophy of the medial temporal lobe, including the hippocampus, in subjects with amnestic MCI in contrast with subjects with multiple domain MCI, which was associated with a more diffuse pattern of cortical atrophy. ${ }^{39}{ }^{40}$ It is difficult to compare our results directly, as these studies used different MRI techniques. In addition, the multiple domain MCI subtype in these studies included both subjects with impairment in multiple non-memory domains and in both memory and nonmemory domains. Our study suggests that the classification of subjects with and without memory impairment into one subtype may result in a heterogeneous group.

The differences in MTA observed across the MCI subtypes were largely attributable to subjects aged over 70 years, suggesting that the concept of MCI subtypes may be less useful in younger subjects. Our observation may be explained by the fact that the prevalence of dementia strongly increases with age ${ }^{41}$ and that in subjects with MCI the risk for developing dementia over a 10 year interval is strongly dependent on age. ${ }^{42}$

$\mathrm{WMH}$ have been reported to be associated with executive function in $\mathrm{MCI}^{43}$ and control subjects. ${ }^{44}$ Therefore, we hypothesised that WMH might be associated with nonamnestic MCI. However, we could not demonstrate a direct association between the severity of $\mathrm{WMH}$ and any of the MCI subtypes, in keeping with a recent study. ${ }^{17}$ Also, the prevalence of vascular risk factors did not differ across the MCI subtypes. This is in contrast with the study of Mariani et al who reported a higher frequency of vascular risk factors and white matter hyperintensities on MRI in their single non-memory MCI group. ${ }^{16}$ The fact that in our study subjects were recruited from memory clinics and that subjects with a history of stroke were excluded from the study may have led to an under-representation of subjects with significant small vessel disease. Furthermore, the absence of controls subjects did not allow us to compare the prevalence of WMH with a normal population.

Although MCI subtypes were similar with regard to WMH severity, differences between combined involvement of MTA and $\mathrm{WMH}$ were observed (fig 2). To our knowledge, this has not been investigated before. The higher prevalence of MTA in the amnestic MCI subtypes compared with the other subtypes was mainly because isolated MTA was more common in amnestic MCI. This suggests that $\mathrm{AD}$ may be the underlying cause in most amnestic MCI patients with MTA. In nonamnestic $\mathrm{MCI}$, isolated MTA was relatively rare as most subjects with MTA also had WMH. As the APOE-e4 allele is a risk factor for $\mathrm{AD}$, we post hoc investigated whether the relation of APOE-4 with MTA was different for subjects with isolated MTA and subjects with MTA and WMH. In the whole sample, the proportion of APOE e4 carriers in the group with isolated MTA was higher $(29(53 \%))$ than that in the group with combined MTA and WMH $(16(33 \%, p=0.05))$. This effect was also present in subjects with non-amnestic MCI: seven (58\%) subjects with isolated MTA were APOE-e4 carrier compared with four (17\%) for subjects with MTA and WMH $(p=0.03)$. This suggests that in subjects with MTA and WMH, MTA may have a different pathophysiological background, in which $\mathrm{AD}$ pathology is possibly less relevant. The group of subjects with subjective complaints has received relatively little attention in previous studies so far, and the clinical outcome of these subjects still remains unclear. ${ }^{45}{ }^{46}$ In our study, the majority of subjects categorised as subjective complaints showed no or few abnormalities on MRI, suggesting that these subjects are in the earliest stages of a neurodegenerative disease or that other factors underlie the complaints.

The cross sectional design of our study limits interpretation about causal mechanisms underlying MRI measures and MCI subtypes. Another limitation may be the fact that neuropsychological test batteries differed across the various sites, although this difference may in part be accounted for by correcting for centre in the analyses. Among the strengths of this study is the large sample size of MCI subjects with available MRI scans. All scans were analysed centrally, which reduced the variability of MRI measures to a large extent. Another strength of this study is its clinical setting, which makes the results relevant for clinical practice.

In conclusion, these data provide evidence that the clinical subtypes of MCI, based on neuropsychological profiles, may have different brain substrates, especially in older subjects. However, the observed differences were small and clearly an overlap of MRI profiles between the MCI subtypes existed. Further longitudinal analysis is needed to reveal the clinical outcome of MCI subtypes in relation to MRI measures, which is important with respect to preventive and possible early therapeutic interventions in MCI.

Author affiliations: ${ }^{1}$ Department of Neurology, Alzheimer Centre, VU Medical Centre, Amsterdam, The Netherlands; ${ }^{2}$ Department of Psychiatry and Neuropsychology, University of Maastricht, The Netherlands; ${ }^{3}$ IRCCS San Giovanni, Laboratory of Epidemiology and Neuroimaging, Brescia, Italy; ${ }^{4}$ Aristotle University of Thessaloniki, Memory and Dementia Centre, 3rd Department of Neurology, G Papanicolaore General Hospital, Thessaloniki, Greece; ${ }^{5}$ Papageorgiou Hospital, Department of Radiology, Thessaloniki, Greece; ${ }^{6}$ Clinical Neurophysiology Service, Department of Endocrinological and Metabolic Sciences, University of Genoa, Genoa, Italy: ${ }^{7}$ NVS Department, Karolinska Institutet, Karolinska University Hospital, Huddinge, Sweden; ${ }^{8}$ Clinical Memory Research Unit, Department of Clinical Sciences, Malmö, Lund University, Sweden; ${ }^{9}$ Department of Geriatric Psychiatry, Zentralinstitut für Seelische Gesundheit, University of Heidelberg, Mannheim, Germany; ${ }^{10}$ Alzheimer Memorial Centre and Geriatric Psychiatry Branch, Dementia and Neuroimaging Section Department of Psychiatry, Ludwig-Maximilian University, Munich, Germany;

${ }^{11}$ University of Kuopio, Department of Neurology, Kuopio University Hospital, Kuopio, Finland; ${ }^{12}$ Department of Clinical Epidemiology and Biostatistics, VU Medical Centre, Amsterdam, The Netherlands; ${ }^{13}$ Department of Radiology, Alzheimer Centre, VU Medical Centre, Amsterdam, The Netherlands

Acknowledgements: The authors would like to thank Professor Tuula Pirttila, University of Kuopio, for clinical examination of the study subjects at University of Kuopio.

Funding: The study was funded by the European Commission within the 5th framework programme (QLRT-2001-2455). The study was partly supported (HS) by EVO grant from Kuopio University Hospital, Kuopio, Finland, 5772720. The European commission funded the study as a concerted action but had no role in study design or analysis of the data.

Competing interests: None.

Ethics approval: The study was approved by the local medical ethics committee in each centre.

Provenance and peer review: Not commissioned; externally peer reviewed.

\section{REFERENCES}

1. Petersen RC, Smith GE, Waring SC, et al. Mild cognitive impairment: clinical characterization and outcome. Arch Neurol 1999;56:303-8.

2. Petersen RC, Doody R, Kurz A, et al. Current concepts in mild cognitive impairment. Arch Neurol 2001;58:1985-92.

3. Whitwell JL, Petersen RC, Negash S, et al. Patterns of atrophy differ among specific subtypes of mild cognitive impairment. Arch Neurol 2007;64:1130-8.

4. Bennett DA, Wilson RS, Schneider JA, et al. Natural history of mild cognitive impairment in older persons. Neurology 2002;59:198-205. 
5. Petersen RC. Mild cognitive impairment as a diagnostic entity. J Intern Med 2004;256:183-94.

6. Visser PJ, Verhey FR, Hofman PA, et al. Medial temporal lobe atrophy predicts Alzheimer's disease in patients with minor cognitive impairment. J Neurol Neurosurg Psychiatry 2002;72:491-7.

7. Rasquin SM, Lodder J, Visser PJ, et al. Predictive accuracy of MCl subtypes for Alzheimer's disease and vascular dementia in subjects with mild cognitive impairment: a 2-year follow-up study. Dement Geriatr Cogn Disord 2005;19:113-19.

8. Alexopoulos $\mathbf{P}$, Grimmer T, Perneczky $R$, et al. Progression to dementia in clinical subtypes of mild cognitive impairment. Dement Geriatr Cogn Disord 2006;22:27-34.

9. Convit A, de Leon MJ, Golomb J, et al. Hippocampal atrophy in early Alzheimer's disease: anatomic specificity and validation. Psychiatr O 1993;64:371-87.

10. Laakso MP, Soininen H, Partanen K, et al. Volumes of hippocampus, amygdala and frontal lobes in the MRI-based diagnosis of early Alzheimer's disease: correlation with memory functions. J Neural Transm Park Dis Dement Sect 1995;9:73-86.

11. Petersen RC, Jack CR Jr, Xu YC, et al. Memory and MRI-based hippocampal volumes in aging and AD. Neurology 2000;54:581-7.

12. Scheltens $\mathbf{P}$, Leys $\mathrm{D}$, Barkhof $\mathrm{F}$, et al. Atrophy of medial temporal lobes on MRI in "probable" Alzheimer's disease and normal ageing: diagnostic value and neuropsychological correlates. J Neurol Neurosurg Psychiatry 1992;55:967-72.

13. Jack CR Jr, Petersen RC, Xu YC, et al. Prediction of AD with MRI-based hippocampal volume in mild cognitive impairment. Neurology 1999;52:1397-403.

14. Korf ES, Wahlund LO, Visser PJ, et al. Medial temporal lobe atrophy on MRI predicts dementia in patients with mild cognitive impairment. Neurology 2004;63:94-100.

15. Geroldi C, Rossi R, Calvagna C, et al. Medial temporal atrophy but not memory deficit predicts progression to dementia in patients with mild cognitive impairment. J Neurol Neurosurg Psychiatry 2006;17:1219-22.

16. Mariani E, Monastero R, Ercolani $\mathrm{S}$, et al. Vascular risk factors in mild cognitive impairment subtypes. Findings from the ReGAl project. Dement Geriatr Cogn Disord 2007; 24:448-56.

17. Bombois S, Debette $S$, Delbeuck $X$, et al. Prevalence of subcortical vascular lesions and association with executive function in mild cognitive impairment subtypes. Stroke 2007; 38:2595-7.

18. Burns JM, Church JA, Johnson DK, et al. White matter lesions are prevalent but differentially related with cognition in aging and early Alzheimer disease. Arch Neurol 2005;62:1870-6.

19. de Leeuw FE, de Groot JC, Achten E, et al. Prevalence of cerebral white matter lesions in elderly people: a population based magnetic resonance imaging study. The Rotterdam Scan Study. J Neurol Neurosurg Psychiatry 2001;70:9-14.

20. Fazekas F, Kleinert $\mathrm{R}$, Offenbacher $\mathrm{H}$, et al. Pathologic correlates of incidental MRI white matter signal hyperintensities. Neurology 1993;43:1683-9.

21. Pantoni L, Garcia JH. Pathogenesis of leukoaraiosis: a review. Stroke 1997:28:652-9.

22. Wolf $\mathbf{H}$, Ecke GM, Bettin $\mathbf{S}$, et al. Do white matter changes contribute to the subsequent development of dementia in patients with mild cognitive impairment? A Iongitudinal study. Int J Geriatr Psychiatry 2000;15:803-12.

23. van der Flier WM, van Straaten EC, Barkhof F, et al. Medial temporal lobe atrophy and white matter hyperintensities are associated with mild cognitive deficits in nondisabled elderly people: the LADIS study. J Neurol Neurosurg Psychiatry 2005; 76:1497-500

24. Launer LJ, Andersen K, Dewey ME, et al. Rates and risk factors for dementia and Alzheimer's disease: results from EURODEM pooled analyses. EURODEM Incidence Research Group and Work Groups. European Studies of Dementia. Neurology 1999;52:78-84.
25. Visser PJ, Verhey FR, Boada M, et al. Development of screening guidelines and clinical criteria for predementia Alzheimer's disease: the DESCRIPA study. Neuroepidemiology 2008;30:254-65.

26. Folstein MF, Folstein SE, McHugh PR. "Mini-mental state". A practical method for grading the cognitive state of patients for the clinician. J Psychiatr Res 1975;12:189-98.

27. Morris JC. The Clinical Dementia Rating (CDR): current version and scoring rules. Neurology 1993;43:2412-14.

28. Lezak ML. Neuropsychological assessment, 3rd edn. New York: Oxford University Press, 1995.

29. Welsh KA, Butters N, Mohs RC, et al. The Consortium to Establish a Registry for Alzheimer's Disease (CERAD). Part V. A normative study of the neuropsychological battery. Neurology 1994;44:609-14.

30. Masur DM, Fuld PA, Blau AD, et al. Distinguishing normal and demented elderly with the selective reminding test. J Clin Exp Neuropsychol 1989;11:615-30.

31. Novelli G. Three clinical tests for the assessment of lexical retrieval and production. Norms from 320 normal subjects. Arch Psicol Neurol Psichiatria 1986;47:477-506.

32. Reitan. Validity of the Trail Making Test as an indication of organic brain damage. Percept Motor Skill 1958;8:271-6.

33. Osterrieth PA. Le test de copie d'une figure complexe: Contribution a l'e'tude de la perception et de la memoire (The test of copying a complex figure: A contribution to the study of perception and memory). Arch Psychol 1944;30:286-350.

34. Carlesimo GA, Caltagirone C, Gainotti G. The Mental Deterioration Battery: normative data, diagnostic reliability and qualitative analyses of cognitive impairment. The Group for the Standardization of the Mental Deterioration Battery. Eur Neurol 1996;36:378-84.

35. Wahlund LO, Barkhof F, Fazekas F, et al. A new rating scale for age-related white matter changes applicable to MRI and CT. Stroke 2001;32:1318-22.

36. Jack CR Jr, Petersen RC, Xu YC, et al. Medial temporal atrophy on MRI in normal aging and very mild Alzheimer's disease. Neurology 1997;49:786-94.

37. Pennanen C, Kivipelto M, Tuomainen S, et al. Hippocampus and entorhinal cortex in mild cognitive impairment and early AD. Neurobiol Aging 2004;25:303-10.

38. Busse A, Bischkopf J, Riedel-Heller SG, et al. Subclassifications for mild cognitive impairment: prevalence and predictive validity. Psychol Med 2003;33:1029-38.

39. Becker JT, Davis SW, Hayashi KM, et al. Three-dimensional patterns of hippocampal atrophy in mild cognitive impairment. Arch Neurol 2006;63:97-101.

40. Bell-McGinty S, Lopez OL, Meltzer CC, et al. Differential cortical atrophy in subgroups of mild cognitive impairment. Arch Neurol 2005;62:1393-7.

41. Lobo A, Launer LJ, Fratiglioni L, et al. Prevalence of dementia and major subtypes in Europe: A collaborative study of population-based cohorts. Neurologic Diseases in the Elderly Research Group. Neurology 2000;54:S4-9.

42. Visser PJ, Kester A, Jolles J, et al. Ten-year risk of dementia in subjects with mild cognitive impairment. Neurology 2006;67:1201-7.

43. Reed BR, Eberling JL, Mungas D, et al. Effects of white matter lesions and lacunes on cortical function. Arch Neurol 2004:61:1545-50.

44. de Groot JC, de Leeuw FE, Oudkerk M, et al. Cerebral white matter lesions and cognitive function: the Rotterdam Scan Study. Ann Neurol 2000;47:145-51.

45. Flicker C, Ferris SH, Reisberg B. A longitudinal study of cognitive function in elderly persons with subjective memory complaints. J Am Geriatr Soc 1993;41:1029-32.

46. Geerlings MI, Jonker C, Bouter LM, et al. Association between memory complaints and incident Alzheimer's disease in elderly people with normal baseline cognition. Am J Psychiatry 1999;156:531-7. 


\section{JNNP}

\section{White matter hyperintensities and medial temporal lobe atrophy in clinical subtypes of mild cognitive impairment: the DESCRIPA study}

L A van de Pol, F Verhey, G B Frisoni, et al.

J Neurol Neurosurg Psychiatry 2009 80: 1069-1074 originally published online June 18, 2009

doi: 10.1136/jnnp.2008.158881

Updated information and services can be found at:

http://jnnp.bmj.com/content/80/10/1069.full.html

These include:

References This article cites 45 articles, 18 of which can be accessed free at: http://jnnp.bmj.com/content/80/10/1069.full.html\#ref-list-1

Article cited in:

http://jnnp.bmj.com/content/80/10/1069.full.htmI\#related-urls

Email alerting Receive free email alerts when new articles cite this article. Sign up in service the box at the top right corner of the online article.

Collections

Articles on similar topics can be found in the following collections

Memory disorders (psychiatry) (1074 articles)

Stroke (1195 articles)

Notes

To request permissions go to:

http://group.bmj.com/group/rights-licensing/permissions

To order reprints go to:

http://journals.bmj.com/cgi/reprintform

To subscribe to BMJ go to:

http://group.bmj.com/subscribe/ 